\title{
MRI based CFD analysis for brain blood flow with IDO-AMR method
}

\author{
T. Aoki ${ }^{1}$, Y. Imai ${ }^{1}$, T. Kobara ${ }^{1} \&$ H. Ikehira ${ }^{2}$ \\ 'Global Scientific Information and Computing Center, \\ Tokyo Institute of Technology, Japan \\ ${ }^{2}$ Informative Molecular Research Section, \\ Department of Medical Imaging,
}

National Institute of Radiological Sciences, Japan

\begin{abstract}
A brain blood structure is extracted from MRI (Magnetic Resonance Imaging) voxel data of a head, and modeled for CFD (Computational Fluid Dynamics) analysis. In order to treat complex structure of the blood vessel with branches, we introduce two major techniques in the blood flow simulation; a high-accurate numerical scheme IDO (Interpolated Differential Operator) which has been developed to solve Navier-Stokes equation as a compact scheme by using local Hermite interpolation, and AMR (Adaptive Mesh Refinement) method to gather fine meshes around the blood flow in Cartesian grid to describe the geometry of the vessel accurately keeping less computation. The computational results show the spatial profile and the temporal change of the velocity and the pressure. It is possible to find the high pressure area in the brain, and the branch ratio of the blood flow for several cases.
\end{abstract}

\section{Introduction}

In the biomechanics, Computational Fluid Dynamics (CFD) is becoming a strong tool for diagnostics in the cardiovascular system. Blood dynamics is of multi-scale physical phenomena and the vessel structure consists of hierarchy branches with complex geometry. Conventional numerical method such as Finite Difference Method is not applicable even if a boundary fitted coordinate is used. 
In moving boundary case, there is a severe problem of mesh generation in Finite Element Method. We use Adaptive Mesh Refinement (AMR) method on Cartesian grid in which fine meshes are introduced adaptively in the region with complex structure. For more realistic study, the elastics of the blood vessel has to be taken into consideration and fluid-structure interaction becomes important.

\section{Blood vessel structure extracted from MRI data}

Recently, high-resolved medical diagnostics images can be obtained from X-ray CT scanning or MRI devices. It is possible to extract the blood vessel structure from the accumulated voxel data. Figure 1 shows the slice image of a head, and it was taken at the MRI device of Department of Medical Imaging, National Institute of Radiological Sciences. We change the transfer function of the volume rendering, and the blood vessel becomes strike in Fig. 2. The blood structure is able to be extracted by connecting the voxel having the same intensity range of the signal.

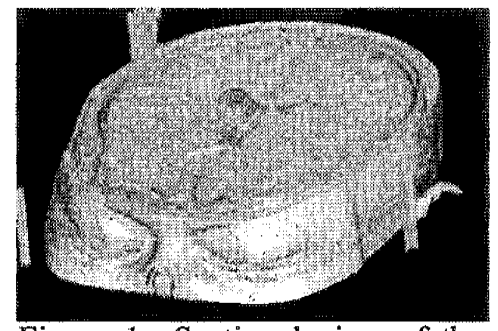

Figure 1: Sectional view of the MRI data of a head.

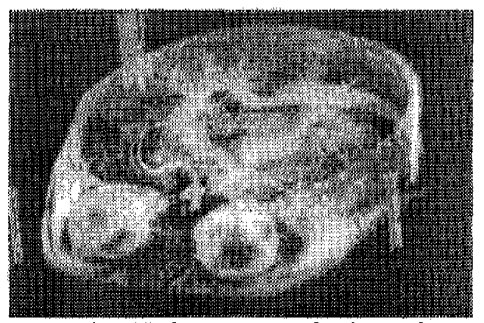

Figure 2: Volume rendering image after the change of transfer function.

Figure 3 shows the vessel structure after one smoothing process however the surface of the vessel is still rough due to the noise mainly coming from the duration time of the diagnosis. It is difficult to use this structure for a numerical simulation as it is, so we propose a modeling for the blood vessel based on the extracted structure. It is assumed that the continuity of the blood vessel and drastic change of the vessel radius is not acceptable.

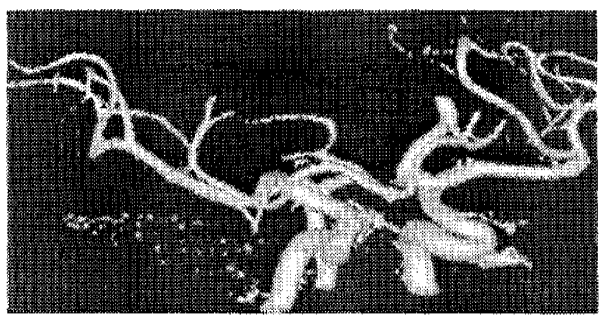

Figure 3: Extracted vessel structure.

The blood vessel structure is modeled by the center lines and the radius as 
illustrated in Fig.4. The process to generate the structure of Fig.4 from the MRI data is not purely automatic. The judgment of the bifurcation and separation of the blood signals from the noise in the narrow vessel compatible with the image resolution has to be done by using the medical knowledge.

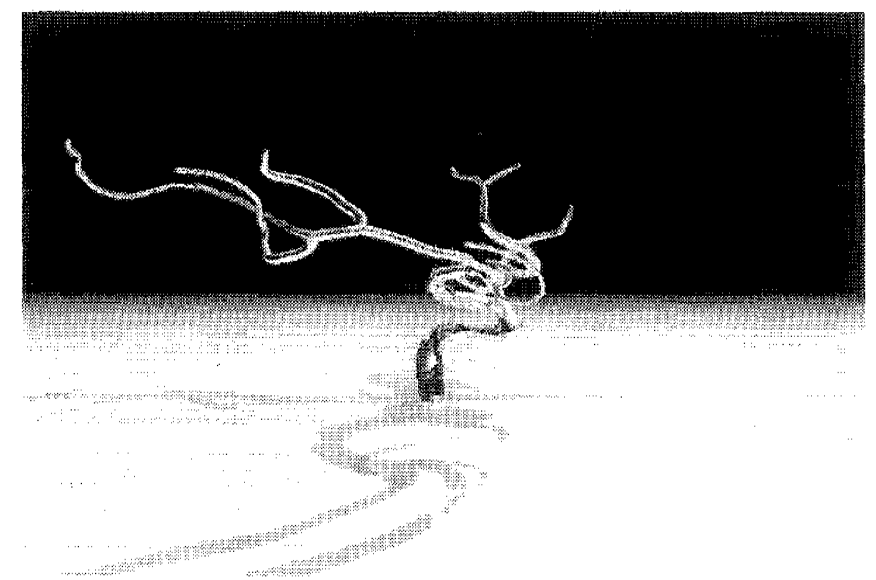

Figure 4: Blood vessel modeling obtained from MRI data.

\section{Numerical Scheme using high accurate interpolation}

We have developed a numerical scheme 'Interpolated Differential Operator (IDO)' for solving hyperbolic, ellipsoidal and parabolic differential equations[1]. The IDO scheme is constructed on the basis of a discretized space (grid points) such as FDM and FEM. The dependent variables defined on the grid have a spatial profile spreading over the local area that covers several grid points. The profile should be an approximate solution of the governing PDE within the area. The IDO scheme requires a spatial interpolation to describe high-order spatial derivatives.

The given equations are solved in the original differential form described by the primitive variables. We regard the derivative terms appearing in the equation as differential operators, and we use the equations derived by temporal and spatial differentiation. We consider the following equation,

$$
f_{t}=\mathfrak{I}_{x} f,
$$

where the subscripts $t$ and $x$ denote the time and spatial derivative operation, respectively. The symbol $\mathfrak{I}_{x}$ stands for a spatial differential operator. By taking time derivatives of eq.(1), we have a series of the equations $f_{t t}=\mathfrak{I}_{x} \mathfrak{I}_{x} f, f_{t t}=\mathfrak{\Im}_{x} \mathfrak{\Im}_{x} \mathfrak{\Im}_{x} f, \cdots$, and so on. In the IDO scheme, we do not use any finite difference expressions, but $\mathfrak{I}_{x}$ and higher derivative terms operate on the interpolation profile $F(x)$ directly.

The IDO scheme has been applied to various fluid problems; compressible Rayleigh-Taylor instability, dendritic solidification, shallow water equations of 
global ocean model, incompressible flow around falling leaves and so on, and good results have been obtained. Several new techniques as Cut-Cell method, Overset grid and Adaptive Mesh Refinement method were introduced in these studies.

In the IDO scheme, the spatial profiles of the dependent variables are obtained by spatial interpolations covering several grid points. It is possible to use various interpolations that can describe accurately the spatial derivatives, and in this paper we use a Hermite interpolation that is determined by matching conditions of both the values and the derivatives. The Hermite interpolation has enough accuracy and compactness, since the local interpolation uses only neighboring grid information. We use two kinds of interpolation whose interpolated domain is different.

Now we consider the interpolation function around the $i$-th grid point in one-dimensional case. The dependent variables $f$ and $f_{x}$ are assumed to be given at all the grid points. In general, numerical information propagates in all the directions, so that the interpolation has to cover the area from $i-1$ to $i+1$. The Hermite interpolation $P(x)$ is obtained by the four matching conditions of $P(-\Delta x)=f_{i-1}, \quad \frac{\partial}{\partial x} P(-\Delta x)=f_{x, t-1}, \quad P(\Delta x)=f_{i+1}$, and $\frac{\partial}{\partial x} P(\Delta x)=f_{x, i+1}$. The coefficients of the fifth-order polynomial (Kondoh, 1995) are determined as follows,

$$
\begin{aligned}
P(x)=a_{c} x^{5} & +b_{c} x^{4}+c_{c} x^{3}+d_{c} x^{2}+f_{x, i} x+f_{i} \\
a_{c} & =-\frac{3}{4 \Delta x^{5}}\left(f_{i+1}-f_{i-1}\right)+\frac{1}{4 \Delta x^{4}}\left(f_{x, i+1}+4 f_{x, i}+f_{x, i-1}\right) \\
b_{c} & =-\frac{1}{2 \Delta x^{4}}\left(f_{i+1}-2 f_{i}+f_{i-1}\right)+\frac{1}{4 \Delta x^{3}}\left(f_{x, i+1}-f_{x, i-1}\right) \\
c_{c} & =\frac{5}{4 \Delta x^{3}}\left(f_{i+1}-f_{i-1}\right)-\frac{1}{4 \Delta x^{2}}\left(f_{x, i+1}+8 f_{x, i}+f_{x, i-1}\right) \\
d_{c} & =\frac{1}{\Delta x^{2}}\left(f_{i+1}-2 f_{i}+f_{i-1}\right)-\frac{1}{4 \Delta x}\left(f_{x, i+1}-f_{x, i-1}\right)
\end{aligned}
$$

The derivatives higher than $f_{x}$ are obtained by taking differentiation of the interpolation function with respect to $x$. At the $i$-th grid point, we have $f_{x x}=\frac{\partial^{2}}{\partial x^{2}} P(0)=2 d_{c} \quad, \quad f_{x x x}=\frac{\partial^{3}}{\partial x^{3}} P(0)=6 c_{c}, \quad f_{x x x x}=\frac{\partial^{4}}{\partial x^{4}} P(0)=24 b_{c} \quad$ and $f_{\text {tox }}=120 a_{c}$, and higher derivatives are zero.

Another interpolation is used for the advection term $u f_{x}$; for example, $f_{t}+u f_{x}=0$. We call this upwind interpolation. When the advection velocity $u$ is positive, the interpolation covers the area from $i$-1 to $i$-th grid. The interpolation function has the following third-order polynomial 


$$
F(x)=a_{u} x^{3}+b_{u} x^{2}+f_{x, j} x+f_{i}
$$

where the coefficients $a_{u}$ and $b_{u}$ are determined by the matching conditions $F(-\Delta x)=f_{i-1}, \frac{\partial}{\partial x} F(-\Delta x)=f_{x, i-1}$, so that we have

$$
\begin{aligned}
& a_{u}=\frac{\left(f_{x, i}+f_{x, i-1}\right)}{\Delta x^{2}}-2 \frac{\left(f_{i}-f_{i-1}\right)}{\Delta x^{3}} \\
& b_{u}=\frac{\left(2 f_{x, i}+f_{x, i-1}\right)}{\Delta x}-3 \frac{\left(f_{i}-f_{i-1}\right)}{\Delta x^{2}} .
\end{aligned}
$$

In the case of $u<0$, the interpolation covers the area from $i$ to $i+1$-th grid point, and the coefficients are derived by the conditions $F(\Delta x)=f_{i+1}, \frac{\partial}{\partial x} F(\Delta x)=f_{x, i+1}$ as follows,

$$
\begin{aligned}
& a_{u}=\frac{\left(f_{x, i}+f_{x, i+1}\right)}{\Delta x^{2}}+2 \frac{\left(f_{i}-f_{i+1}\right)}{\Delta x^{3}} \\
& b_{u}=-\frac{\left(2 f_{x, i}+f_{x, i+1}\right)}{\Delta x}-3 \frac{\left(f_{i}-f_{i+1}\right)}{\Delta x^{2}} .
\end{aligned}
$$

The derivatives higher than $f_{x}$ are obtained by taking derivative of the interpolation function as $f_{x x, i}=\frac{\partial^{2}}{\partial x^{2}} F(0)=2 b_{u}$ and $f_{x x x, i}=\frac{\partial^{3}}{\partial x^{3}} F(0)=6 a_{u}$ at the $i$-th grid point, and higher derivatives more than $f_{\mathrm{xxx}}$ are set to be zero.

In the IDO scheme, the derivative terms included in partial differential equations are classified into advection term and non-advection term, and the above two interpolations are applied selectively.

\section{4-th order SMAC Algorithm}

In order to solve incompressible Navier-Stokes equation, we adapt SMAC algorithm. First, we solve the following equation, that is, the Navier-Stokes equation without the pressure gradient term.

$$
\frac{\partial u}{\partial t}+\boldsymbol{u} \cdot \nabla \boldsymbol{u}=\frac{1}{R e} \Delta \boldsymbol{u}
$$

The current velocity $\boldsymbol{u}^{\mathrm{n}}$ is advanced to $\boldsymbol{u}^{*}$ by the following 4-stages Runge-Kutta method.

$$
\begin{aligned}
& K_{1}=\frac{\partial \boldsymbol{u}}{\partial t}\left(\boldsymbol{u}^{n}, \boldsymbol{u}_{x}^{n}\right), \quad K_{x 1}=\frac{\partial \boldsymbol{u}_{x}}{\partial t}\left(\boldsymbol{u}^{n}, \boldsymbol{u}_{x}^{n}\right), \\
& K_{2}=\frac{\partial \boldsymbol{u}}{\partial t}\left(\boldsymbol{u}^{n}+\frac{1}{2} K_{1} \Delta t, \boldsymbol{u}_{x}^{n}+\frac{1}{2} K_{x 1} \Delta t\right), \quad K_{x 2}=\frac{\partial \boldsymbol{u}_{x}}{\partial t}\left(\boldsymbol{u}^{n}+\frac{1}{2} K_{1} \Delta t, \boldsymbol{u}_{x}^{n}+\frac{1}{2} K_{x 1} \Delta t\right), \\
& K_{3}=\frac{\partial \boldsymbol{u}}{\partial t}\left(\boldsymbol{u}^{n}+\frac{1}{2} K_{2} \Delta t, \boldsymbol{u}_{x}^{n}+\frac{1}{2} K_{x 2} \Delta t\right), \quad K_{x 3}=\frac{\partial \boldsymbol{u}_{x}}{\partial t}\left(\boldsymbol{u}^{n}+\frac{1}{2} K_{2} \Delta t, \boldsymbol{u}_{x}^{n}+\frac{1}{2} K_{x 2} \Delta t\right),
\end{aligned}
$$




$$
\begin{gathered}
K_{3}=\frac{\partial \boldsymbol{u}}{\partial \boldsymbol{t}}\left(\boldsymbol{u}^{n}+K_{2} \Delta t, \boldsymbol{u}_{x}^{n}+K_{x 2} \Delta t\right), \quad K_{x 3}=\frac{\partial \boldsymbol{u}_{x}}{\partial t}\left(\boldsymbol{u}^{n}+K_{2} \Delta t, \boldsymbol{u}_{x}^{n}+K_{x 2} \Delta t\right), \\
\boldsymbol{u}^{*}=\frac{K_{1}+2 K_{2}+2 K_{3}+K_{4}}{6} \Delta t, \quad \boldsymbol{u}_{x}^{*}=\frac{K_{x 1}+2 K_{x 2}+2 K_{x 3}+K_{x 4}}{6} \Delta t
\end{gathered}
$$

The intermediately advanced $\boldsymbol{u}^{*}$ and $\boldsymbol{u}_{x}^{*}$ are corrected by the pressure gradient term so as to satisfy the incompressible condition $\nabla \cdot u=0$, as follows.

$$
u^{n+1}=u^{*}-\nabla p \cdot \Delta t, \quad \quad u_{x}^{n+1}=u_{x}^{*}-\nabla p_{x} \cdot \Delta t
$$

The pressure used in the above equation is obtained by solving the Poisson equation,

$$
\Delta p=\frac{\nabla \cdot u^{*}}{\Delta t} .
$$

The 5-th center interpolation is applied to the derivative terms in eq.(6). In order to show the accuracy of the Poisson solver of IDO scheme, one-dimensional Poisson equation $f_{x x}=\rho$ is examined, where $\rho$ is the source term. For the dependent variable $f$, the IDO scheme solves the original Poisson equation $F_{x x}=\rho$, For another dependent variable $f_{x}$, we use the additional equation $F_{x x x}=\rho_{x}$ derived by taking differentiation of the original equation, and $F$ is the interpolation function. The explicit descriptions of the discretized formula are written down as follows,

$$
\begin{aligned}
& \frac{2}{\Delta x^{2}}\left(f_{i+1}-2 f_{i}+f_{i-1}\right)-\frac{1}{2 \Delta x}\left(f_{x, i+1}-f_{x, i-1}\right)=\rho_{i}, \\
& \frac{15}{2 \Delta x^{3}}\left(f_{i+1}-f_{i-1}\right)-\frac{3}{2 \Delta x^{2}}\left(f_{x, i+1}+8 f_{x, i}+f_{x, i-1}\right)=\rho_{x, i} .
\end{aligned}
$$

In the case of $\rho=\sin (k x)$ for $0 \leq x \leq L$ and $k=4 \pi / L$, we estimate the numerical error by the formula

$$
\sigma^{2}=\sum_{i=1}^{N}\left(\frac{\sin \left(k x_{i}\right)}{k^{2}}-f_{i}\right)^{2} \text {, }
$$

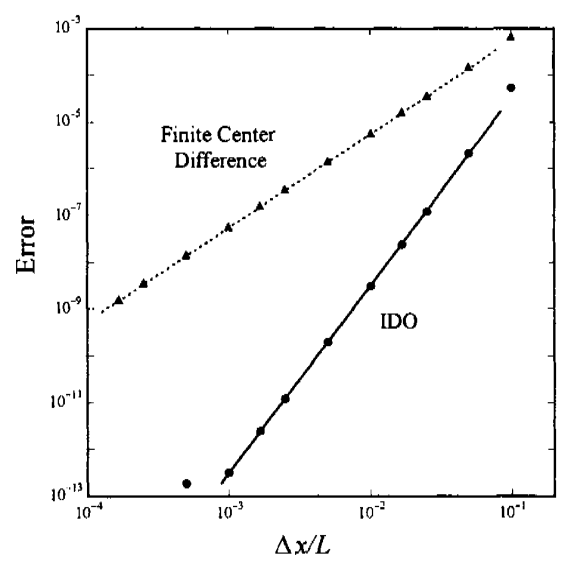


Figure5: Accuracy of the result of Poisson equation by IDO scheme. The triangle markers indicate the result obtained by the center finite difference method. The solid circles representing IDO scheme shows fourth order spatial accuracy.

where $N$ is the total grid number and the grid interval $\Delta x=L /(N-1)$. The boundary conditions at $x=0(i=1)$ and $x=L \quad(i=N)$ are set to be $f_{1}=f_{N}=0$ and $f_{x, 1}=f_{x, N}=k^{-1}$, respectively. The circles in Fig. 1 show the average error $\sigma$ of the IDO scheme with the center interpolation as a function of $\Delta x$ for the given domain area $0 \leq x \leq L$, and it is found that the error has a scaling of $\Delta x^{4}$. As a reference, the results of the finite difference scheme $\left(f_{i+1}^{-} 2\right.$ $\left.f_{i}+f_{i-1}\right) / \Delta x^{2}=\rho_{i}$ are plotted by triangular marks, and it has $\Delta x^{2}$ scaling. It is understood that the IDO scheme has fourth order spatial accuracy, since the formula $F_{x x}$ derived from the center interpolation uses five values of the grid without $f_{x, i}$ according to eq.(7). In the expression of $F_{x x x}, f_{i}$ is not included.

When we consider extending the IDO scheme to multi-dimensional one, it is natural that we introduce the first order spatial derivative in each dimensional direction. In the two-dimensional case, the dependent variables $f, f_{x}$ and $f_{y}$ are required at least, however additionally we introduce $f_{x y}$ for the purpose to interpolate $f_{x}$ in the $y$-direction and to interpolate $f_{y}$ in the $x$-direction. The introduction of $f_{x y}$ closes a series of the equation self-consistently. Similarly, we use a set of the 8 dependent variables $f, f_{x}, f_{y}, f_{z}, f_{x y}, f_{x z}, f_{y z}$ and $f_{x y z}$, and it is necessary to solve 8 equations to determine these variables [2]. The above Poisson equation is solved by SOR method, and the convergence of the iteration procedure is almost same as the second order finite difference case, and it is possible to accelerate it by multi-grid method [3].

\section{Pulsatile Flow into stenosis channel}

AS a preliminary check of the blood flow simulation, we study the incoming flow from the left inlet into the stenosis channel shown in Fig.6 with the waveform of two pulsatile flows of Fig.7 at a Reynolds number $\mathrm{Re}=750$ and a Strouhal number $\mathrm{St}=0.024$. We use a Cartesian uniform mesh in order to construct the boundary condition for the blood vessel. The computation is done on the same condition as Ref.[4].

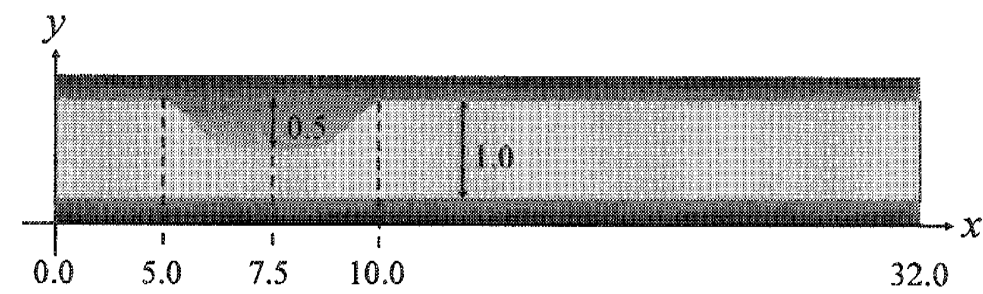

Figure 6: Schematic view of the computational geometry. 


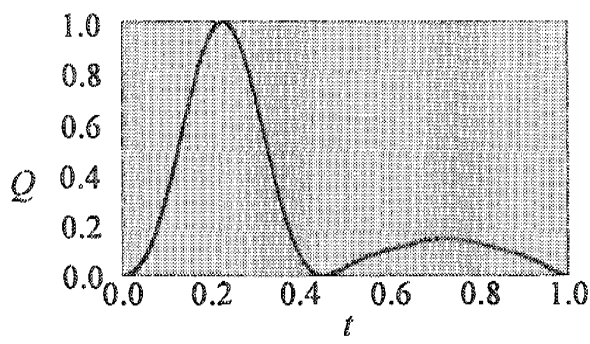

Figure 7: Waveform of the incoming flow

The snap shots of the vortex profile are shown in Fig.8 (a) - (h). With decrease of the incoming flow after the time $t=0.4$, the pressure at the stenose goes down so that the vortex are generated in the downstream. These computational results are in good agreement with that of the reference [4].

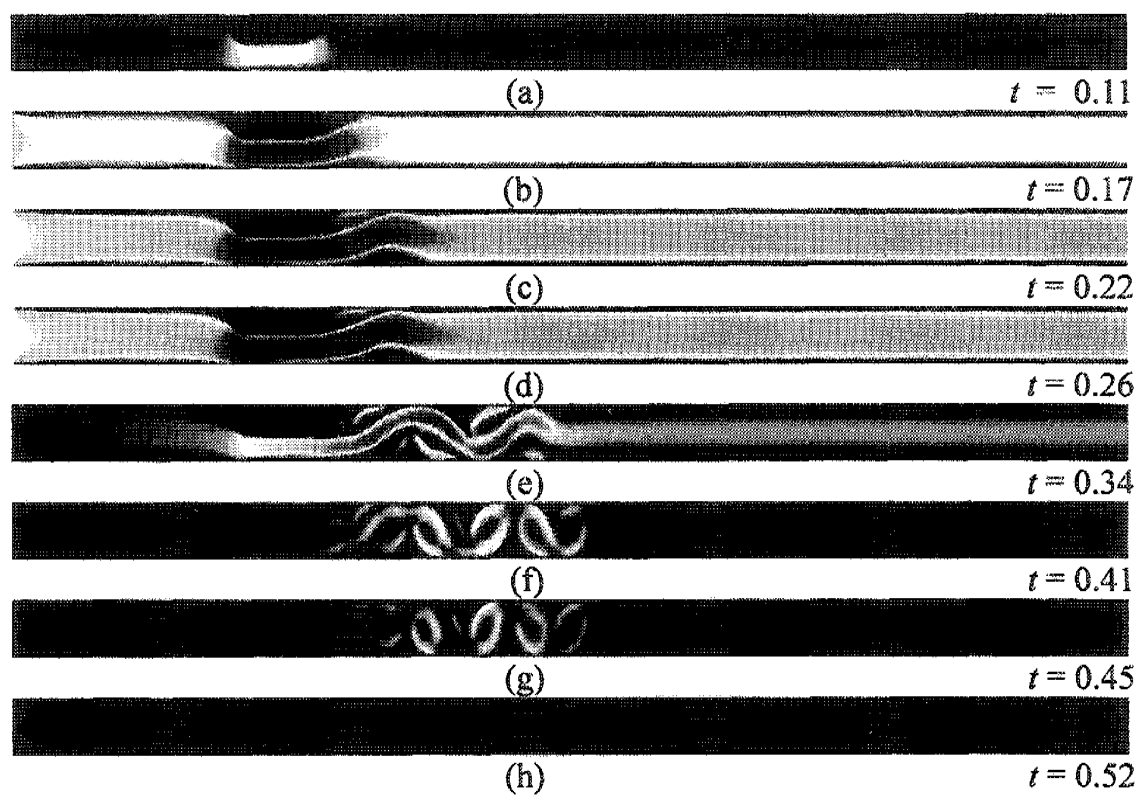

Figure 8: Vortex contours at $\operatorname{Re}=750$ and $\mathrm{St}=0.024$ with two pulsatile incoming flow. (a) $t=0.11$, (b) $t=0.17$, (c) $t=0.22$, (d) $t=0.26$, (e) $t=0.34$, (f) $t=0.41$, (g) $t=0.45$, (h) $t=0.52$.

\section{Adaptive Mesh Refinement Approach}

A simple uniform Cartesian grid is not suitable for blood flow simulations, because blood flow region is located with narrow tube structure. From the view 
point of fluid-structure interaction, Cartesian grid is suitable for moving boundary problems. In the previous work, we introduce Cut-Cell method to describe a complex shape on Cartesian grid [5] however there is the resolution limit to treat a small-size structure. In order to satisfy these two requests, we introduce Adaptive Mesh Refinement (AMR) method [6] to the blood flow analysis to keep a high resolution only around the blood vessel.

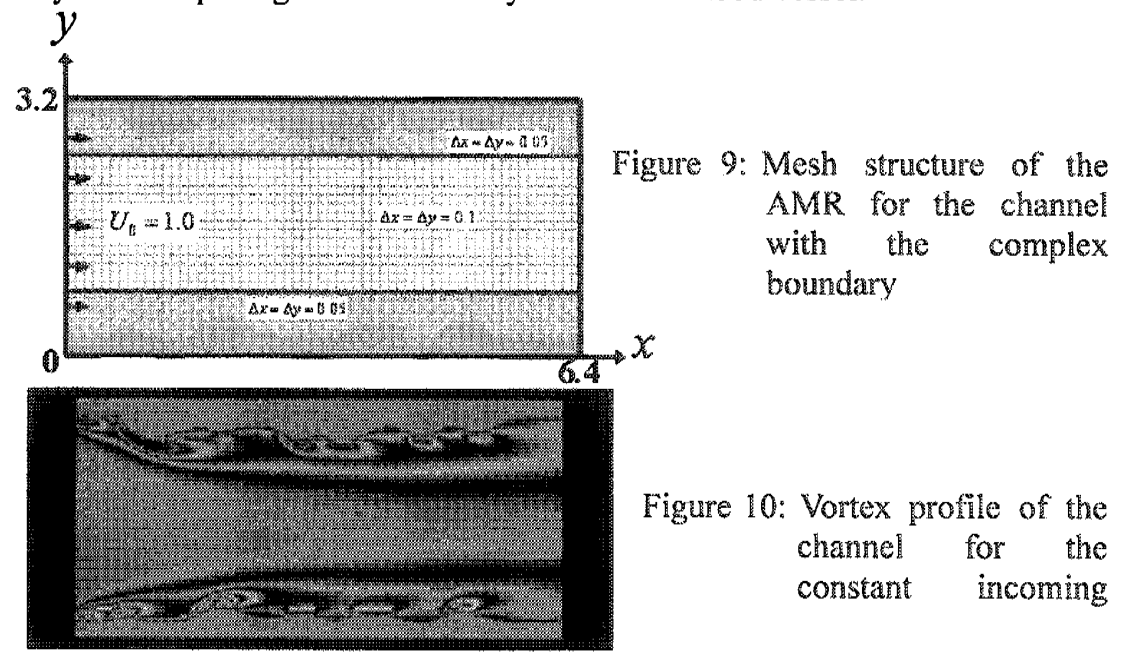

Figure 9 shows the grid structure to describe the complex boundary shape, and the fine meshes are located near the boundary. The vortex profile of the computational result in the channel for the constant incoming flow at $\operatorname{Re}=200$ is shown in Fig.10. More realistic channel flow for the blood vessel is modeled in 2-dimentional case and shown in Fig.11. Fine meshes are located at the branch regions to keep high accuracy at the points.

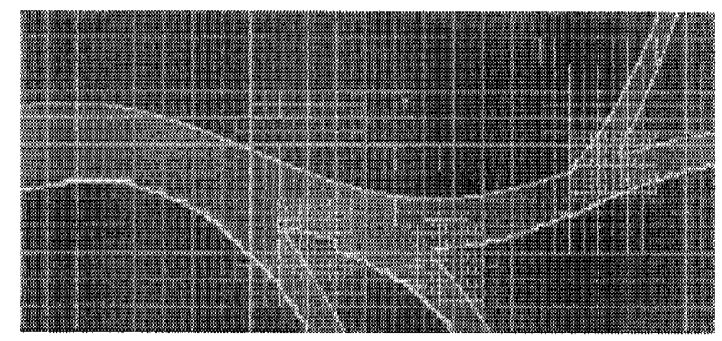

Figure 11: Mesh structure for 2-dimensional channel with branches.

The AMR method requires high accurate interpolation at the connection between different resolution meshes, and the Hermite interpolation works very well. The IDO scheme can solve Navier-Stokes equation in the non-uniform mesh as $1: 2$ grid distance ratio. Figure 12 indicates the profile of the flow speed for $\operatorname{Re}=350$ 
after the flow reaches the steady state. It is found that the branch ratio of the flow in the complex tube structure with branches.

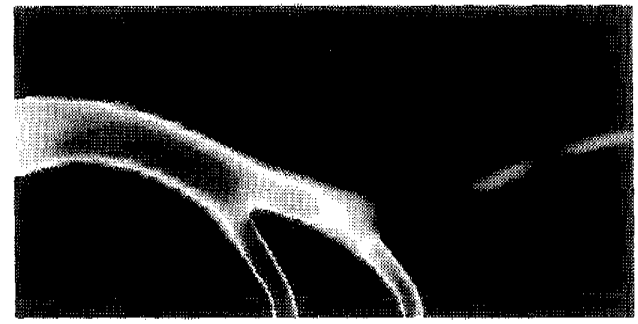

Figure 12: Velocity profile of the flow with branches.

\section{Conclusions}

A brain blood structure is extracted from MRI (Magnetic Resonance Imaging) voxel data of a head, and modeled for CFD (Computational Fluid Dynamics) analysis. By using AMR method, it is possible to describe fine structures with less memory consuming and treat moving boundary problems. IDO (Interpolated Differential Operator) scheme can solve Navier-Stokes equation accurately in compatible with AMR method. The preliminary checks of our method are done and give good computational results. We have a perspective to extend to three-dimensional case.

\section{References}

[1] T. Aoki, Comp. Phys. Comm., 102 [1-3] (1997) 132-146.

[2] T. Aoki, Comp. Fluid Dynamics J., 4 [3] (1995) 279-291.

[3] T. Aoki, Nishita, S and Sakurai, K., Comp. Fluid Dynamics J., 9 [4] (2001) 406-417.

[4] H. Liu, and T. Yamaguchi, ASME J. Biomech. Eng., 123 (2001) 88-96.

[5] T. Aoki, Comp. Phys. Comm., 142 [1-3] (2001) 326-329

[6] S. B. Baden, et al., Structured Adaptive Mesh refinement (SAMR) Grid Methods, Springer (2000). 